\title{
Efficacy of a technology-based, integrated smoking cessation and alcohol intervention for smoking cessation in adolescents: Results of a cluster-randomised controlled trial
}

\author{
Haug, Severin ; Paz Castro, Raquel ; Kowatsch, Tobias ; Filler, Andreas ; Schaub, Michael P
}

DOI: https://doi.org/10.1016/j.jsat.2017.09.008

Posted at the Zurich Open Repository and Archive, University of Zurich

ZORA URL: https://doi.org/10.5167/uzh-140313

Journal Article

Accepted Version

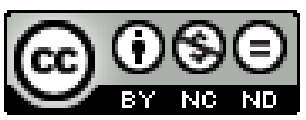

The following work is licensed under a Creative Commons: Attribution-NonCommercial-NoDerivatives 4.0 International (CC BY-NC-ND 4.0) License.

Originally published at:

Haug, Severin; Paz Castro, Raquel; Kowatsch, Tobias; Filler, Andreas; Schaub, Michael P (2017). Efficacy of a technology-based, integrated smoking cessation and alcohol intervention for smoking cessation in adolescents: Results of a cluster-randomised controlled trial. Journal of Substance Abuse Treatment, 82:55-66.

DOI: https://doi.org/10.1016/j.jsat.2017.09.008 
Efficacy of a technology-based, integrated smoking cessation and alcohol intervention for smoking cessation in adolescents: Results of a cluster-randomised controlled trial

Severin Haug*1, Raquel Paz Castro ${ }^{1}$, Tobias Kowatsch ${ }^{2}$, Andreas Filler $^{2}$, Michael P Schaub ${ }^{1}$

*Corresponding author

${ }^{1}$ Swiss Research Institute for Public Health and Addiction at Zurich University, Konradstrasse 32,

8031 Zurich, Switzerland

${ }^{2}$ Institute of Technology Management, University of St. Gallen, Dufourstrasse 40a, 9000 St.

Gallen, Switzerland

E-Mails:

Severin Haug: severin.haug@isgf.uzh.ch

Raquel Paz Castro: raquel.paz@isgf.uzh.ch

Tobias Kowatsch: tobias.kowatsch@unisg.ch

Andreas Filler: afiller@ethz.ch

Michael P Schaub: michael.schaub@isgf.uzh.ch 


\begin{abstract}
Objective: To test the efficacy of a technology-based integrated smoking cessation and alcohol intervention versus a smoking cessation only intervention in adolescents. Methods: This was a two-arm, parallel-group, cluster-randomised controlled trial with assessments at baseline and six months follow-up. Subjects in both groups received tailored mobile phone text messages to support smoking cessation for 3 months, and the option of registering for a program incorporating strategies for smoking cessation centred around a self-defined quit date. Subjects in the integrated intervention group also received tailored feedback regarding their consumption of alcohol and, for binge drinkers, tailored mobile phone text messages encouraging them to maintain their drinking within low-risk limits over a 3-month period. Primary outcome measures were the 7-day point prevalence of smoking abstinence and change in cigarette consumption. Results: In 360 Swiss vocational and upper secondary school classes, 2,127 students who smoked tobacco regularly and owned a mobile phone were invited to participate in the study. Of these, 1,471 (69.2\%) participated and 6-month follow-up data were obtained for 1116 (75.9\%). No significant group differences were observed for any of the primary or secondary outcomes. Moderator analyses revealed beneficial intervention effects concerning 7-day smoking abstinence in participants with higher versus lower alcohol consumption. Conclusions: Overall, the integrated smoking cessation and alcohol intervention exhibited no advantages over a smoking cessation only intervention, but it might be more effective for the subgroup of adolescent smokers with higher alcohol consumption. Providing a combined smoking cessation and alcohol intervention might be recommended for adolescent smokers with higher-level alcohol consumption.
\end{abstract}

Keywords: tobacco, alcohol, mobile phone, text messaging, adolescents 


\section{Introduction}

Tobacco smoke is a major contributor to the global burden of disease (GBD 2013 Risk Factors Collaborators, 2015). Although the prevalence of tobacco smoking among adolescents in developed countries has been falling over the last two decades, smoking continues to be a serious problem, particularly among those with lower education levels (Inchley et al., 2016; The ESPAD group, 2015). In Switzerland, $29 \%$ of male and $21 \%$ of female adolescents between the ages of 15 and 19 years smoke cigarettes either daily or occasionally (Gmel, Kuendig, Notari, \& Gmel, 2016).

Despite knowledge of several environmental, societal and individual factors, like tobacco control policies, friends and peers smoking, alcohol use, and nicotine dependence, which influence adolescent smoking and smoking cessation (Black \& Chung, 2014; Tworek et al., 2010; van Zundert \& Engels, 2009; Van Zundert, Kuntsche, \& Engels, 2012), evidence on the effectiveness of smoking cessation interventions for adolescents is limited (Stanton \& Grimshaw, 2013; Sussman \& Sun, 2009). The 2013 Cochrane Review of smoking cessation interventions for those younger than 20 years identified 28 trials, of which only three achieved statistically-significant results (Stanton \& Grimshaw, 2013). Although the authors concluded that interventions incorporating elements sensitive to stage of change, motivational enhancement, and cognitive behavioural therapy are promising, they also (1) claimed that there currently was insufficient published empirical evidence for them to support any particular interventional model; and (2) argued for future randomized controlled trials that were both methodologically-robust and sufficiently-powered.

Use of the Internet and mobile phones is extremely popular among adolescents and young adults. For example, in 2016 in Switzerland, 99\% and 95\% of adolescents ages 12-19 owned a mobile phone and used the Internet at least daily, respectively (Waller, Willemse, Genner, Suter, \& Süss, 2016). As such, these two media platforms have the potential to provide smoking cessation support to the vast majority of adolescents and young adults. Moreover, mobile phone text 
messaging opens the door to both individualized and interactive information delivery that is readily accessible everywhere, and irrespective of time. A Cochrane review of 12 studies on primarily text messaging-based smoking cessation interventions revealed a beneficial impact of such interventions on six-month cessation outcomes (Whittaker, McRobbie, Bullen, Rodgers, \& $\mathrm{Gu}, 2016)$.

A meta-analysis on 14 studies addressing text messaging interventions for adolescent and young adult alcohol or tobacco use (Mason, Ola, Zaharakis, \& Zhang, 2015) indicated that effect sizes varied but appeared to cluster in the small to medium range. A recent study from the US (Mason et al., 2016) tested the efficacy of a text messaging smoking cessation intervention to engage urban African-American adolescents through an automated texting program utilizing motivational interviewing-based peer network counselling. At 6-months follow up, participants receiving the intervention significantly decreased the number of days they smoked cigarettes and the number of cigarettes they smoked per day, compared to an attention control intervention.

Another text messaging-based program for smoking cessation that primarily focused on adolescents was tested among vocational school students in Switzerland. This program — named SMS-COACH - considered the participants` intention to quit according to the Health Action Process Approach (HAPA) (Schwarzer et al., 2007). During an online assessment, smoking behaviour and attitudes towards smoking cessation were assessed. Thereafter, subjects received one text message every week to evaluate their targeted smoking behaviours for the duration of the three-month intervention. They also received two feedback messages weekly, which were tailored both to their baseline data and their responses to the weekly SMS assessments. Seven-day rates for smoking abstinence at the six-month follow-up evaluation were $12.5 \%$ and $9.6 \%$ in the intervention and control group, respectively. Though this difference was not statisticallysignificant, relative to their control-group counterparts, those in the intervention group did experience a significantly-greater reduction in the mean daily number of cigarettes they smoked from baseline to follow-up (Haug, Schaub, Venzin, Meyer, \& John, 2013). Despite the promising 
results of this text messaging-based program, the rate of abstinence from cigarettes did not increase.

One way to potentially enhance smoking cessation rates among those seeking to do so would be to combine smoking cessation and alcohol reduction, for several reasons. First, the vast majority of people who smoke cigarettes also drink alcohol, and adolescent and young adult smokers often engage in hazardous drinking. For example, in one Swiss study assessing vocational school students, $81.3 \%$ of smokers, but only $48.5 \%$ of non-smokers drank hazardously (Haug, Schaub, Salis Gross, John, \& Meyer, 2013).

Second, cravings for cigarettes generally increase during alcohol consumption, as do relapses after successful smoking cessation (Kahler, Spillane, \& Metrik, 2010; Sayette, Martin, Wertz, Perrott, \& Peters, 2005). This has been confirmed for adolescent smokers (Van Zundert et al., 2012). A third reason to suggest that integrated program targeting both smoking cessation and alcohol reduction might have merit stems from two different pilot studies involving young adults. In both these studies, which compared an integrated smoking-alcohol intervention and an intervention aiming for smoking reduction alone, the former was associated with clinically-relevant, albeit statistically non-significant superiority in smoking abstinence at final follow-up. In the first of these pilot studies, involving 41 young adult smokers who regularly engaged in binge drinking, tobacco abstinence rates after 12 weeks of treatment were substantially higher (36\% vs. $21 \%$ ) among those in the integrated-intervention group (Ames et al., 2010). In the second, somewhatlarger pilot study involving 95 young adults, corresponding end-of-treatment abstinence rates were 21\% and 9\% (Ames, Pokorny, Schroeder, Tan, \& Werch, 2014).

Hence, the current paper compares the efficacy, in adolescents, of two technology-based programs: (1) an optimized version of the former $S M S-C O A C H$ program, now called MobileCoach Tobacco (MCT), which targets smoking cessation alone; and (2) an expanded program, called MobileCoach Tobacco+ $(M C T+)$, which integrates smoking cessation and alcohol reduction into one intervention. To our knowledge, ours is the first sufficiently-powered 
randomized controlled trial (RCT) to compare an integrated intervention targeting both smoking cessation and alcohol reduction, and a smoking cessation only intervention.

\section{Materials and methods}

\subsection{Study objectives and design}

This two-arm, parallel-group, cluster-randomised controlled trial, which used school class as the randomisation unit, was designed to evaluate the efficacy of a technology-based integrated smoking cessation and alcohol intervention relative to a smoking cessation only intervention, in terms of inducing adolescents to stop smoking. The study was registered at Current Controlled Trials ISRCTN (ISRCTN02427446, assigned 8 September 2014) and conducted in Switzerland, where participants were recruited between September 2014 and July 2016. The 6-month follow-up assessments were conducted between March 2015 and January 2017, and the study protocol was published on 5 November 2014 (Haug, Paz Castro, et al., 2014).

Our main hypothesis was that the integrated intervention would be more effective than the smoking cessation only intervention at reducing cigarette consumption and achieving smoking abstinence. Secondary outcome measures assessed at the 6-month follow-up evaluation included: (1) 30-day point prevalence of smoking abstinence; (2) stage of change, as per the Health Action Process Approach (HAPA) (Schwarzer, 2008); (3) any attempts to quit over the 6-month observation period; and (4) quantity of alcohol consumption. Furthermore, the study aimed at investigating socio-demographic and health-related moderators of the interventions' efficacy. The study protocol was approved by the ethics committee of the Faculty of Philosophy at the University of Zurich, Switzerland (date of approval: 13 August, 2014), and the trial conducted in compliance with the Declaration of Helsinki. The study was implemented as described in the published study protocol (Haug, Paz Castro, et al., 2014), with the following modification: to adequately consider the nested data structure among students in classes (intra-class correlation for the primary outcome was $13.5 \%$ and $3.7-9.5 \%$ for secondary outcomes), we performed 
generalized linear mixed modelling (GLMM; Laird \& Ware, 1982) rather than conventional regression models.

\subsection{Participants, setting, and procedure}

The assessment involved vocational school students, due to the high prevalence of smoking in this population (approximately $42 \%$ of one such Swiss sample were daily or occasional tobacco smokers, Haug, Schaub, Salis Gross, et al., 2013). Prevention specialist centres in the Swiss cantons of Zurich, Basel, Berne, Lucerne and Zug invited vocational schools to participate in a study examining the efficacy of a web- and text messaging-based programme designed to support smoking cessation. Twenty-four vocational schools, incorporating 360 classes in total, agreed to participate in the study.

Study assistants (Psychology graduate students or employees drawn from the prevention specialist centres) invited all students in the participating classes to take part in an online health survey during a regular school lesson reserved for health education. They also informed students that some of them would be invited to participate further in a study testing the efficacy of an intervention for health promotion. To reduce reporting bias, the study assistants provided no further information regarding the purpose of the study before screening was complete. Online screening included data collection on demographics, tobacco smoking status, physical activity, body weight, alcohol consumption, and mobile phone ownership. Inclusion criteria for the intervention trial were: (1) daily or occasional cigarette smoking (at least 4 cigarettes over the preceding month and at least one cigarette within the preceding week) and (2) ownership of a mobile phone.

Eligible individuals were informed about data protection, the aim of the study, the assessments, and reimbursement. Research assistants provided study and programme information online and on paper. Eligible individuals were informed that they could withdraw from participation at any time by sending a text message expressing this intention. To optimize participation, a small 
compensation of 10 Swiss francs was offered for participation in the study at both the baselineand follow-up assessment. Moreover, participants were offered a compensation of 0.5 Swiss francs for responding to each of the 11 weekly SMS assessments conducted during the program. Once they had signed a formal informed-consent form, all participants were asked to provide their mobile phone number, invited to choose a username, and directed towards a baseline assessment of past and present smoking history and intentions. This included questions on intentions to quit, daily/weekly cigarette use, previous cessation attempts, number of friends who smoke, age at smoking onset, smoking cessation outcome expectancies, scenarios in which craving for cigarettes typically occur, alternative strategies to handle these craving situations, and the cost per pack of cigarettes. Those in the integrated smoking cessation and alcohol intervention group also were asked questions about their typical drinking days and times, information we believed necessary for us to appropriately tailor their intervention's content.

For the 6-month follow-up assessment, computer-assisted telephone interviews were conducted by trained interviewers (Psychology graduate students).

\subsection{Randomisation and Concealment of Group Allocation}

To avoid spill-over within classes, a cluster-randomised controlled trial design was used, with school class set as the randomisation unit. Because there was such heterogeneity (e.g., related to gender and profession) in the students between the different vocational school classes, stratified randomisation was done, creating separate randomisation lists for each school. To ensure approximately equal sample sizes in the study groups, a block randomisation procedure was performed using computer-generated, randomly-permuted blocks of four school classes, as described elsewhere (Pocock, 1994).

Research assistants supervising the baseline assessment in the vocational schools were blinded to the group allocation of school classes. In addition, group allocation was not revealed to participants until they had provided their informed consent, username, mobile phone number, and 
baseline data. Research assistants who performed the computer-assisted follow up assessments for primary and secondary outcomes also were blinded to subject group allocation.

\subsection{Sample Size Calculation}

Effect size was estimated using the results of two previous studies, one an RCT and the other a pilot study. In the RCT, which assessed the smoking cessation efficacy of a program called SMS$\mathrm{COACH}$ among vocational school students, the 7 -day point prevalence rate was roughly $12 \%$ at six months of follow-up in the intervention group (Haug, Schaub, Venzin, et al., 2013). In the pilot study, which evaluated the efficacy of an integrated smoking cessation and binge-drinking intervention, roughly a 50\% increase in smoking abstinence rate was documented relative to standard treatment (Ames et al., 2010). Using these data, we assumed seven-day point prevalence abstinence rates of 18 and $12 \%$ in the integrated intervention and smoking cessation only group, respectively, at six months of follow-up. To detect this difference $(18-12 \%=6 \%), \mathrm{n}=588$ in each study group would provide $80 \%$ power and $95 \%$ confidence for 2 -sided Pearson chi-square analysis, using G-Power software (Faul, Erdfelder, Lang, \& Buchner, 2007). To further adjust for vocational school students being nested within classes, we adopted a potential design effect of 1.15 (with an assumed number of participants per school class of $n=4$; and an intra-cluster correlation coefficient of 0.05 (Haug, Schaub, Venzin, et al., 2013)). As such, final per-group and total-study estimates for sample size were $\mathrm{n}=675$ and $\mathrm{N}=1350$, respectively.

\subsection{Intervention}

\subsubsection{Technological background}

The two interventions compared in this study, $M C T$ and the $M C T+$, both were created using the MobileCoach system, which is described elsewhere (Haug, Kowatsch, Castro, Filler, \& Schaub, 2014). The MobileCoach system's source code is available as an open-source project at 
http://mobile-coach.eu. To guarantee subject privacy and data transfer security, both password protection and Secure Sockets Layer encoding were used.

\subsubsection{Theoretical background}

The smoking cessation intervention is primarily based on the Health Action Process Approach (HAPA) (Schwarzer, 2008). This health behaviour model distinguishes between the various motivation processes that result in goal setting (individuals in this stage are called 'pre-intenders') and the volition processes that lead to the health behaviour of interest. According to this theory of health change, individuals are subdivided into three groups: (1) those who remain inactive, who are called 'pre-intenders'; (2) those who are taking steps towards the desired behaviour, who are called intenders; and those who have already adopted the desired behaviour, who are called 'actors'.

Several socio-cognitive factors are believed to have meaningful roles promoting an intention to act. These include individual's self-perceived advantages or benefits of smoking cessation and disadvantages or risks of further smoking (together called 'outcome expectancies), as well as the person's perception of personal risk, and their perceived self-efficacy during the initial preintentional stage. Once someone has entered the intentional stage, planning processes are crucial to achieving the desired action and, once that action has been initiated, self-regulatory skills are crucial to maintaining the desired health behaviour. One example would be learning strategies to cope with cravings. Additionally, elements borrowed from the Social Norms Approach (Perkins, 2003) were integrated into the person's current intervention, as were so called 'if this-then that' plans, which link situational cues with responses that effectively achieve desired outcomes (Gollwitzer \& Sheeran, 2006).

The web-based component of the alcohol intervention included normative feedback based on the social norms approach (Perkins, 2003). The text messaging-based portion of the alcohol intervention primarily relied on the following socio-cognitive constructs from major psychological 
models of health behaviour change like social cognitive theory (McAlister, Perry, \& Parcel, 2008) and the HAPA (Schwarzer, 2008): outcome expectations, motivation to drink within low-risk limits, self-efficacy, and planning processes.

\subsubsection{Overview of the interventions' components}

Once subjects completed the baseline survey, those assigned to the $M C T+$ group receiving the combined intervention and those assigned to the $M C T$ smoking cessation-only group both received individually-tailored mobile phone text messages to support smoking cessation for a 3-month period; and both were offered the option of registering for a more intensive program with strategies for smoking cessation centred around a self-defined quit date. However, those in the $M C T+$ group also received individually-tailored web-based feedback on their drinking behaviours, relative to a reference group representing age and gender norms; and one weekly text message that encouraged them to restrict their alcohol consumption to within low-risk limits for a three-month period, if they had reported binge drinking at baseline

The rationale for providing the web-based normative feedback in the MCT+ group for all participants but the separate text messages to restrict drinking within low-risk limits solely to binge drinkers was based on theoretical considerations and previous results on potential iatrogenic effects of alcohol interventions for non-problem drinkers (Werch \& Owen, 2002). Presenting accurate information about peer group norms in a credible way is conjectured, in line with the social norms approach (Perkins, 2003), to reduce both problem drinkers' and non-drinkers' personal perceptions of peer pressure to consume large quantities of alcohol. Similarly, the few available studies on normative feedback interventions (Bertholet et al., 2015; Prince, Reid, Carey, \& Neighbors, 2014) showed no increase in alcohol use among lighter drinkers or subjects who did not report problem drinking, compared to control group participants. Rather, these studies indicated protective short-term effects of these normative feedback interventions. However, potential iatrogenic effects of other intervention elements, e.g., addressing outcome expectancies 
or protective behavioural strategies for non-problem drinkers could not be ruled out (Werch \& Owen, 2002).

The text messages were typically 150-200 characters long, some of which included web links to thematically-appropriate video clips, pictures, and/or websites.

\subsubsection{Web-based feedback on drinking behaviours (MCT+)}

Web-based feedback was given to participants in the $M C T+$ group as soon as they finished their baseline assessment, the feedback content having been extracted from effective intervention programs primarily developed for college and university students in the USA and Canada (Cunningham, Humphreys, Kypri, \& van Mierlo, 2006; Doumas, McKinley, \& Book, 2009), then modified for the target group of German-speaking adolescents in Switzerland, ages 16-20, with varied educational backgrounds. Age- and gender-specific norms for alcohol consumption were drawn from a previous study (Gmel, Venzin, Marmet, Danko, \& Labhart, 2012) which had examined the frequency of binge drinking, alcohol volume, and the maximum number of drinks consumed on a single occasion in 973 vocational and upper secondary school students in the Canton of Zurich, Switzerland. The web-based feedback included individually-tailored graphic and textual information on (1) the number of drinks consumed weekly, relative to age and genderspecific reference groups; (2) money spent on drinking; (3) the calorie count of consumed alcoholic drinks; and (4) the frequency of binge drinking relative to age- and gender-specific reference groups.

\subsubsection{Text messages stimulating drinking within low-risk limits (MCT+)}

Only subjects in the $M C T+$ group who reported binge drinking at baseline - defined as consuming, on a single occasion, five or more drinks for men and four or more drinks for women within the previous month — received one weekly text message encouraging them to restrict drinking to within low-risk limits for a 3 month-period. The timing of this text message alternated 
biweekly: one week on Saturday at $7 \mathrm{pm}$, and the next week at that particular individual's most typical day and time for heavy drinking (e.g., Friday at $10 \mathrm{pm}$ ).

The text messages provided information on (1) strategies for drinking within low-risk limits; and

(2) the association between smoking and alcohol consumption, particularly regarding the importance of avoiding or restricting alcohol to minimal amounts to successfully achieve smoking abstinence among those participants who either intend to quit smoking or have already quit. For example, a message might read: 'Hey Martin! Did you know that that drinking alcohol has been proven to increase a person's cigarette cravings? Remember this when you go out next time and try drinking little or no alcohol. It will make it easier for you to stay cigarette free.'

\subsubsection{Text messages to support smoking cessation (MCT+ and MCT)}

Throughout the three-month intervention period, subjects in both intervention groups received one text message prompt every week that either assessed smoking-related target behaviours or encouraged them to participate in a quiz or message contest. All they needed to do to answer to these prompts was type a single letter, number or sentence using the mobile phone's reply function. Each weekly SMS prompt was sent at a fixed time (Tuesday at $6 \mathrm{pm}$ ). The content of the prompt depended upon the individual's Health Action Process Approach (HAPA) stage of change, as well as whether the prompt was delivered on an odd- or even-numbered week of the intervention week.

Smoking-related target behaviour included the HAPA stage of change, assessed every four weeks through the subject's response to the question — 'Have you recently smoked cigarettes?' — for which the following response options were provided: (1) 'Yes, and I do not intend to quit' (preintender); (2) 'Yes, but I am considering quitting' (pre-intender); (3) 'Yes, but I seriously intend to quit' (intender); and (4) 'No, I quit smoking' (actor). Every four weeks we also, among preintenders, asked about the number of cigarettes smoked per day or week (depending on smoking status: daily/occasionally). Meanwhile, for intenders and actors, we asked about whether their 
individually-chosen strategies to cope with craving situations, assessed within the baseline assessment, had been applied. For example: 'Did you apply the following strategy recently? When I am at a party, I distract myself from smoking by dancing. Yes (Y) No (N) '.

Participants received an immediate feedback message after responding to prompts regarding smoking-related target behaviours. For example: 'You can be really proud of yourself! Since the last assessment, you've smoked about 4 fewer cigarettes per day. That means you're on the right path towards an active and healthy lifestyle.'

Forty-eight hours after this prompt (Thursday at 6pm) they received an additional message tailored to their current HAPA stage of change and individual data that they had provided during their baseline assessment. Pre-intenders received text messages providing information (1) on the risks of smoking; (2) on the benefits of smoking cessation; and (3) regarding methods to improve their ability to successfully stop smoking. Intenders and actors received text messages providing information (1) on how to use individual resources for quitting (e.g., social support); (2) on how to overcome barriers to smoking cessation (e.g., friends who smoke, and stress); and (3) ways to enhance their ability to successfully stop smoking. Sample text messages for the different stages of change are displayed in Figure 1.

A quiz was conducted thrice during the intervention period. It included questions concerning: (1) smoking norms (percentage of smokers within the subject's age- and gender-specific reference group); (2) health consequences of smoking cessation (days until positive health consequences are realized); and (3) personal expenditures on cigarettes (money spent for cigarettes per year, see also Figure 1). Participants received immediate individualized feedback on their answer. If they did not respond within 48 hours, they were sent the correct response.

Twice within the intervention period, a contest was conducted that required participants to either send in a motivational text message to help other participants quit smoking (for intenders; see Figure 1) or suggest concrete ways to help others quit smoking (for intenders and actors). Fortyeight hours later, the best text message from each of the two categories — which were rated 
weekly by a tobacco cessation expert from the Swiss Research Institute for Public Health and Addiction — was distributed anonymously to participants in the respective category.

\subsubsection{Optional additional text-messages for smoking cessation (MCT+ and MCT)}

Participants who intended to quit smoking (intenders and actors) were offered the option of receiving additional text messages for quit-day preparation and relapse prevention. Participants in these stages were informed about this option biweekly. After subjects entered their intended quit date, the program provided two daily text messages in weeks -1 through +1 , followed by one daily text message in weeks +2 and +3 . For example: 'Good evening, John. Since tomorrow's going to be your first day smoke-free, why don't you throw all your cigarettes, ash trays, and lighters away today! Stay active tomorrow and be sure to have some chewing gum on hand.'

\subsection{Assessments and outcomes}

\subsubsection{Baseline measures}

The screening assessment included questions on the following demographic characteristics: subject gender, age, and immigration background. We asked about each student's parents' country of birth to identify potential immigrants. Based on this information, participants were assigned to one of the following categories: (1) neither parent born outside Switzerland; (2) one parent born outside Switzerland; or (3) both parents born outside Switzerland. Although this information provided no reliable indicator for race or ethnicity, the presence of a migrant background in a German speaking country was strongly associated with poorer lexical-grammatical skills (Melzer, Rissling, \& Petermann, 2015), which might influence receptivity of the interventions.

The following health-related characteristics were assessed: physical activity, body weight, typical weekly alcohol consumption, and maximal alcohol consumption over the previous month. Selfreported moderate to vigorous physical activity was measured by a question derived from the Health Behaviour in School Aged Children (HBSC) study (Currie, Nic Gabhainn, \& Godeau, 2009): 
"Outside school, how many hours per week do you exercise or participate in sports that make you sweat or out of breath?"

Quantity of alcohol consumed was assessed via a 7-day drinking calendar similar to the Daily Drinking Questionnaire (Collins, Parks, \& Marlatt, 1985), for which participants were asked to think about a typical week in the preceding month and record the number of standard drinks they typically consumed each day during that week. Examples of standard drinks containing 12-14 g of ethanol were provided for beer, wine, spirits, alcopops, and cocktails, along with conversion values (e.g., three $0.5 \mathrm{~L}$ cans of beer $=6$ standard drinks). Maximal alcohol consumption was assessed by asking participants to report the number of standard drinks consumed on their heaviest drinking occasion over the preceding 30 days.

Tobacco smoking status was assessed by asking the question — "Are you currently smoking cigarettes?" - with the following response options: (1) Yes, I smoke cigarettes daily; (2) Yes, I smoke cigarettes occasionally, but not daily; and (3) No. In occasional smokers, we also assessed the number of days they smoked in a typical month, as well as the total number of cigarettes they had smoked over the past seven days. In daily smokers and occasional smokers who smoked at least four cigarettes over the preceding month and at least one cigarette over the preceding week, we assessed the following additional smoking-related variables: mean number of cigarettes smoked per day; HAPA stage of change; and the number of previous attempts to quit.

In daily smokers, we asked about the number of cigarettes smoked on a typical day. In occasional smokers, we initially asked about the typical number days they smoked each month; and, later, the number of cigarettes they smoked on a typical smoking day. For occasional smokers, the average number of cigarettes smoked per day was calculated by multiplying the typical number of smoking days per month by the number of cigarettes smoked on a typical smoking day, and dividing this by 30 days. The HAPA stage of change (Lippke, Ziegelmann, Schwarzer, \& Velicer, 2009), was assessed by asking — "Have you recently smoked cigarettes?" — with the following available response options: (1) "Yes, and I do not intend to quit" (Pre-contemplation), (2) "Yes, but I am 
considering quitting" (Contemplation), or (3) "Yes, but I seriously intend to quit" (Preparation). They also were asked about prior attempts to quit with the question — "Have you ever made a serious attempt to quit smoking?" — with the response options: "No", "Yes, once", and "Yes, more than once".

\subsubsection{Program participation and usage}

To evaluate each subject's level of program acceptance, we analysed log files of the text messaging system in which all incoming and outgoing text messages were recorded. The number of responses to the weekly text message prompts and the number of program participants who unsubscribed from the program (program attrition) were examined. At follow-up, we assessed text message usage by asking participants whether they (1) read through their messages thoroughly; (2) took only a short look at their messages; or (3) did not read their messages. Furthermore, program participants were asked to indicate whether the programme had influenced their smoking behaviours or not.

\subsubsection{Follow up measures}

The following outcome variables were assessed at the six-month follow up: (1) smoking status; (2) 7-day and (3) 4-week smoking abstinence; (4) mean number of cigarettes smoked per day; (5) HAPA stage of change; (6) number of attempts to quit within the past six months; and (7) level of alcohol consumption. The main outcome criteria were 7-day point prevalence of smoking abstinence and the mean number of cigarettes smoked per day.

With respect to smoking status, subjects were asked to indicate whether they smoked (1) daily or (2) occasionally, or (3) had stopped smoking. We also calculated seven-day and four-week point prevalence rates for smoking abstinence (not having smoked a puff within the past seven days and four weeks prior to follow-up, respectively) [23]. Among daily smokers, we evaluated the number of cigarettes smoked on a typical day. For occasional smokers, we assessed the typical number of smoking days per month and number of cigarettes smoked on a typical smoking day, and calculated 
the mean daily number of cigarettes smoked, as described previously. For participants who claimed not to smoke anymore, the value for their daily number of cigarettes smoked was set at zero.

Each subject's HAPA stage of change was assessed using a question similar to that asked at baseline. Participants claiming that they no longer smoked were assigned to the Action stage. Attempts to quit made within the previous six months were asked about using the yes/no question — "Have you made a serious attempt to quit smoking within the previous six months?" It was presumed that all those who no longer smoked had made a serious attempt to quit. Quantity of alcohol consumed was assessed as at baseline using a 7-day drinking calendar for which participants were asked to think about a typical week over the preceding month and record the number of standard drinks they typically consumed each day during that week.

The primary outcome measures assessed at the 6-month follow-up were: (1) 7-day point prevalence of smoking abstinence; and (2) the mean number of cigarettes smoked per day. Secondary outcome measures, included: (1) 30-day point prevalence of smoking abstinence; (2) HAPA stage of change (Schwarzer, 2008); (3) any attempts to quit over the 6-month observation period; and (4) level of alcohol consumption.

\subsection{Data analysis}

We initially examined the data for outliers, based on self-reported numbers of standard drinks per week and cigarettes per day, which were entered as free text. Baseline differences between participants in the study groups were identified by Pearson chi-square analysis for categorical variables, and either by Student's t tests or Mann-Whitney U tests for continuous variables that were normally and non-normally distributed, respectively. The same tests were applied to examine whether or not participants lost to follow up differed from those who responded, as a function of study group.

The intra-class correlation coefficients for the primary and secondary outcomes ranged from 3.7 to $13.5 \%$. Therefore, intervention effects for binary outcomes were tested using generalized linear 
mixed modelling (GLMM), while intervention effects for continuous outcomes were analysed using linear mixed modelling (LMM). Analysis of binary outcomes focused on follow-up values, whereas analysis of continuous outcomes focused on differences between baseline and follow-up values. The independent variable was the treatment group (fixed effect), and a single random effect was modelled for school class (random intercept).

Further ancillary analyses were calculated to detect moderators of the two interventions' efficacy. For this, all interactions between socio-demographic or health-related variables (see Table 1) and treatment group were included separately in the GLMM or LMMs. Moderation analyses were estimated only for the primary outcomes and only for one interaction at a time. All analyses were based on a complete-case (CC) and intention-to-treat (ITT) dataset.

For ITT analyses, we used multiple imputation procedures as described elsewhere (Van Buuren, 2012). Missing at random (MAR) was assumed, since missingness in study variables was associated with measured covariates. Overall predictors of missingness were age and the number of text messages answered by the participant; thus, they were incorporated in all the imputation models for the study outcomes. A specific predictor of missing data at follow-up by study group was smoking status, which was also included in the imputation models. The remaining study outcome predictors were chosen based on their association with these outcomes (correlation coefficient $r>0.30$ ). Group condition was included in all outcome imputations to preserve any potential interventional effect. Additionally, school class was included in the imputation model to account for the clustered structure of data. For binary variables, we used logistic regression; for categorical variables, multinomial logit models; and for continuous variables, predictive mean matching (Van Buuren, 2012). After inspecting 40 imputed datasets revealed no systematic bias in convergence, the final inferences were derived from this solution.

All analyses were conducted with and without controlling for the following baseline differences: age, migration and physical activity. Within the results section, the unadjusted values were reported, unless the adjusted results differed in either direction, magnitude or significance. 
Analyses were performed using SPSS version 23 and R version 3.2.1 via the lme4 (Bates, Maechler, Bolker, \& Walker, 2014) and mice (Buuren \& Groothuis-Oudshoorn, 2011) packages. Results with a Type I error rate of $p<.05$ in two-sided tests were considered statistically significant.

\section{Results}

\subsection{Study participation}

Participants' progression through the trial is depicted in Figure 2. Within 360 school classes, 5'694 students were present at the time of the online screening assessment. Of these, 2' 127 (37.4\%) met the inclusion criteria, and 1'471 (69.2\%) agreed to participate and completed the baseline health survey. Out of the 360 classes, 167 classes containing 741 subjects were randomly assigned to the single intervention (MCT), while 174 classes containing 730 subjects were assigned to the combined intervention (MCT+). Four hundred and ninety-five binge drinkers $(67.8 \%)$ among the 730 subjects in the MCT+ group received the web-based feedback on drinking behaviours and text messages stimulating drinking within low-risk limits, whereas 235 subjects $(32.2 \%)$ in the MCT+ group, who did not report binge drinking within the previous month, solely received the former. Follow-up assessments were completed by $556(75.0 \%)$ participants in the MCT group and by 560 (76.7\%) participants in the MCT+ group.

\subsection{Programme attrition, usage and acceptance}

During the text messaging-based programme, which lasted for 12 weeks, 13 of the $741(1.8 \%)$ participants in the single intervention unsubscribed, compared to 18 of the $730(2.5 \%)$ participants in the combined intervention $\left(\chi^{2}=0.90, p=.34\right)$. The mean number of replies to the weekly SMS text message assessments was 6.4 out of $11(S D: 3.6)$ in the MCT group versus 6.7/11 (3.5) in the $\mathrm{MCT}+\operatorname{group}(t=-1.37, p=.17)$. 
Of 1088 participants with valid follow-up data, 1052 (96.7\%) indicated that they had received text messages regularly. Almost all of them $(n=940,89.4 \%)$ reported that they had 'read the SMS messages thoroughly', of which $89.3 \%$ (467) were in the MCT group and $89.6 \%$ (473) in the MCT+ group. Only 9.1\% (96) reported that they only 'took a quick look at the SMS messages' (MCT: $9.0 \%$ vs. MCT+: 9.3\%), while 1.4\% (15) indicated that they 'did not read the SMS messages' (MCT: $1.7 \%$ vs. MCT+: $1.1 \%)\left(\chi^{2}=0.66, d f=2, p=.72\right)$. Within the MCT group, $47.8 \%$ (250) of the subjects affirmed that the programme had influenced their smoking behaviours, compared to $48.3 \%$ (254) within the MCT + group $\left(\chi^{2}=0.03, p=.87\right)$.

\subsection{Sample characteristics}

Baseline characteristics for the study sample are shown in Table 1. Baseline differences between the two groups were detected for age $(t=-2.89, p<.01)$ immigration background $\left(\chi^{2}=8.54, p=\right.$ $.014)$, and physical activity $(U=-3.08, p<.01)$. Participants in the MCT + group generally were younger, more often had parents who had both been born in Switzerland and were more physically active than those in the single intervention group.

Concerning attrition bias, analysis revealed that participants in the MCT+ group who were lost to follow up were more likely to report a lower weight $(t=2.15, d f=353, p=.034)$ and occasional smoking at baseline $\left(\chi^{2}=4.32, d f=1, p=.038\right)$ than those lost to follow-up in the MCT group.

\subsection{Primary outcome analysis}

Table 2 presents 7-day point prevalence rates for smoking abstinence and the pre-to-post intervention differences in the number of cigarettes smoked daily for both study groups. No significant inter-group differences were observed for either primary outcome. The 7-day smoking abstinence rate at follow-up was $13.9 \%$ in the MCT group and $15.0 \%$ in the MCT+ group (CC: $\mathrm{p}=.61$, ITT: $\mathrm{p}=.82$ ). The mean number of cigarettes smoked per day decreased from baseline to 
follow-up by 2.8 versus 2.7 in the MCT versus MCT+ group, respectively (CC: p=.97, ITT: $\mathrm{p}=.93)$. The adjusted results did not differ from the unadjusted results.

\subsection{Secondary outcome analysis}

Table 3 summarizes the secondary outcomes in both study groups. Neither CC nor ITT analyses revealed any significant inter-group differences in the secondary outcomes. The adjusted results did not differ from the unadjusted results.

\subsection{Moderation analysis}

Using the $\mathrm{CC}$ dataset, separate moderation analyses of the 7-day point prevalence of smoking abstinence revealed gender, smoking status, severity of tobacco use, drinks consumed per week, and the maximum number of drinks consumed on a single occasion to be influential. On ITT inferential testing, however, only the maximum number of drinks consumed on a single occasion remained as a significant predictor of 7-day point prevalence of smoking abstinence. The combined intervention was more effective among subjects who reported a higher maximum number of drinks consumed on a single occasion at baseline than among those who reported a lower maximal number of drinks (CCA: $z=2.64, p=.008$; ITT: $z=1.964, p=.049$ ) (Figure 3). In subjects who reported higher-risk drinking (maximum number of drinks consumed on a single occasion at baseline $>75$ percentile, i.e. $>9$ drinks), the combined intervention increased the percentage of subjects who did not smoke within the last 7 days from $9.5 \%$ to $16.5 \%(+7.0 \%)$, whereas it was similar $(+0.2 \%)$ effective in participants who reported lower-risk drinking (maximum number of drinks consumed on a single occasion at baseline $<25$ percentile, i.e. $<3$ drinks) and those reporting a medium level of risk-drinking (-2.6\%, maximum number of drinks consumed on a single occasion at baseline between percentiles 25 and 75, i.e. 3-9 drinks). Using CC, separate moderation analyses of reductions in cigarettes smoked per day, the number of drinks consumed per week was found to influence intervention success; however, this moderation 
was only tendentiously but not statistically significantly using the ITT data $(\mathrm{CCA}: \mathrm{t}=-2.62, \mathrm{p}=$ $.008 ;$ ITT: $\mathrm{t}=-1.956, \mathrm{p}=.051)$. Subjects in the combined intervention group who reported higher weekly alcohol use at baseline decreased their cigarette use to a larger extent than those in the single intervention group.

\section{Discussion}

The primary aim of this study was to compare the efficacy of an Internet- and mobile phone delivered integrated smoking cessation and alcohol intervention against that of a smoking cessation only intervention among adolescents. Four main findings were revealed: (1) The majority of students accepted some intervention, with 7 out of 10 participating in the programme and associated study. (2) Programme attrition and acceptance did not differ between participants receiving the integrated versus smoking cessation only intervention. (3) Across the total sample, the integrated smoking cessation and alcohol intervention exhibited no beneficial, but also no detrimental effects relative to the smoking cessation only intervention, with respect to any of the primary or secondary outcomes. (4) Among the subgroup of adolescent smokers with high-risk alcohol consumption, moderation analysis indicated that the integrated intervention might be more effective at supporting smoking cessation.

The proactive invitation for program participation in combination with the offer of a low-threshold technology-based intervention allowed us to convince roughly 7 of 10 smoking students (69\%) to participate in the program. Taking into account that $86 \%$ of the program participants indicated no serious intention to quit at baseline, this high participation rate is of special relevance. Within a Swedish study, more than 200,000 university students were invited to participate in a text messaging-based smoking cessation intervention via email, but fewer than 2.000 students completed the assessments and could be included within the trial. Consistent with studies on telephone- or physician-delivered advice for adolescent smoking cessation, our results underscore 
the importance of proactive recruitment strategies and low-threshold interventions to attain high participation rates (Heffner et al., 2016; Pbert et al., 2015).

The ease of use and flexibility of SMS text messaging to send and receive messages at any time and place, as well as the potential to receive individually-tailored information, might be responsible for the high use and retention rates identified in this study, which are comparable to a previous study on text-messaging-based smoking cessation counselling in this target group and setting (Haug, Schaub, Venzin, et al., 2013). Within both study groups (MCT, MCT+), nearly all program participants $(98.2 \%, 97.5 \%)$ remained logged in until the end of the 3-month program; and 89.3 and $89.6 \%$, respectively, indicated that they had read the text messages thoroughly. Therefore, we did not find any indications that the additional intervention components within the integrated intervention affected program use or acceptance. Similar rates and ratings were identified for session attendance and intervention helpfulness, respectively, in a pilot study that compared an integrated smoking cessation and binge-drinking intervention versus standard smoking cessation therapy in young adult smokers (Ames et al., 2014).

On the other hand, our findings regarding the relative efficacies of the integrated and standard intervention are contrary to those obtained in previous pilot studies conducted in the United States (Ames et al., 2014; Ames et al., 2010), which found higher, albeit not statistically significant, smoking abstinence rates within smokers who underwent the integrated binge-drinking and smoking cessation intervention. One main difference between these pilot studies and our own concerns our inclusion of smokers without hazardous drinking, who we included based on the hypothesis and previous results that adolescents without hazardous drinking also might benefit from social norm interventions intended to reduce hazardous drinking (Doumas \& Hannah, 2008; Haug et al., 2017; Perkins, 2002). Another recently-published study revealed that smokers with non-problematic drinking benefitted from text messaging-based interventions to reduce problem drinking, while non-smokers did not (Paz Castro, Haug, Kowatsch, Filler, \& Schaub, 2017). However, concerning smoking cessation, the results of our moderation analyses indicate that, 
among smokers without hazardous drinking, the integrated intervention was not particularly beneficial, though it appeared to be more beneficial than the smoking-only intervention among smokers who drank more heavily. Unfortunately, our study lacked the statistical power to test this hypothesis, meaning that studies with larger subject samples or more stringent inclusion criteria, thereby including only smokers with hazardous drinking, still need to be conducted.

The main limitation of the current study was its reliance on self-report and the associated possibility that the results could have been influenced by social desirability. One example of this is smoking status, which was never biochemically verified; subjects merely were asked. On the other hand, it is reasonable to expect that any potential over-reporting of smoking abstinence would be independent of the intervention administered. Moreover, in its recommendations, the Society for Research on Nicotine and Tobacco suggests that scenarios exist wherein any added accuracy gained by biological validation is offset such that its use becomes unnecessary and, perhaps, even undesirable (SRNT Subcommittee on Biochemical Validation, 2002). Measures we used to minimize the under- or over-reporting of alcohol consumption included the assurance of confidentiality and anonymous assessments conducted via tablet computers without personal contact, which may have increased the reliability of self-reported data.

Further study limitations include (1) the lack of an assessment-only control group, which precludes any conclusions about the absolute effectiveness of the interventions, (2) that several contextual factors which can influence smoking and cessation have not been assessed and controlled for, e.g., peers smoking, family members smoking, exposure to tobacco advertising, (3) that the provision of the additional text messages stimulating drinking within low risk limits for binge drinkers only, within the combined intervention, makes it difficult to disentangle the moderator effect; (4) the relatively short follow-up period, with only one assessment six months subsequent to the baseline assessment; and (5) limited generalizability, because we used a convenience sample of school classes willing to participate in the study. It also is feasible that 
study participation and subject retention rates were influenced by the compensation every subject received for study and program participation.

\section{Conclusions}

The results of this study suggest that offering adolescent smokers a technology-based integrated smoking cessation and alcohol intervention, irrespective of their alcohol use, has no overall beneficial effects compared to an intervention targeting smoking cessation only. On the other hand, among the subgroup of adolescent smokers who consume larger quantities of alcohol on single occasions, the integrated intervention might be more effective than the alternative at inducing smoking cessation, though further, adequately-powered trials that only include smokers with higher levels of alcohol use remain necessary to adequately test this hypothesis.

\section{Acknowledgments}

Funding for this study was provided by the Swiss Tobacco Prevention Fund (No. 13.006402).

\section{References}

Ames, S. C., Pokorny, S. B., Schroeder, D. R., Tan, W., \& Werch, C. E. (2014). Integrated smoking cessation and binge drinking intervention for young adults: a pilot efficacy trial. Addictive Behaviors, 39(5), 848-853.

Ames, S. C., Werch, C. E., Ames, G. E., Lange, L. J., Schroeder, D. R., Hanson, A. C., \& Patten, C. A. (2010). Integrated smoking cessation and binge drinking intervention for young adults: a pilot investigation. Annals of Behavioral Medicine, 40(3), 343-349.

Bates, D., Maechler, M., Bolker, B. M., \& Walker, S. (2014). Fitting linear mixed-effects models using lme4. Journal of Statistical Software (http://arxiv.org/abs/1406.5823). 
Bertholet, N., Cunningham, J. A., Faouzi, M., Gaume, J., Gmel, G., Burnand, B., \& Daeppen, J. B. (2015). Internet-Based Brief Intervention to Prevent Unhealthy Alcohol Use among Young Men: A Randomized Controlled Trial. PloS One, 10(12), e0144146.

Black, J. J., \& Chung, T. (2014). Mechanisms of change in adolescent substance use treatment: how does treatment work? Substance Abuse, 35(4), 344-351.

Buuren, S., \& Groothuis-Oudshoorn, K. (2011). mice: Multivariate imputation by chained equations in R. Journal of Statistical Software, 45(3).

Collins, R. L., Parks, G. A., \& Marlatt, G. A. (1985). Social determinants of alcohol consumption: the effects of social interaction and model status on the self-administration of alcohol. Journal of Consulting and Clinical Psychology, 53(2), 189-200.

Cunningham, J. A., Humphreys, K., Kypri, K., \& van Mierlo, T. (2006). Formative evaluation and three-month follow-up of an online personalized assessment feedback intervention for problem drinkers. Journal of Medical Internet Research, 8(2), e5.

Currie, C., Nic Gabhainn, S., \& Godeau, E. (2009). The Health Behaviour in School-aged Children: WHO Collaborative Cross-National (HBSC) study: origins, concept, history and development 1982-2008. International Journal of Public Health, 54 Suppl 2, 131-139.

Doumas, D. M., \& Hannah, E. (2008). Preventing high-risk drinking in youth in the workplace: a web-based normative feedback program. Journal of Substance Abuse Treatment, 34(3), 263-271.

Doumas, D. M., McKinley, L. L., \& Book, P. (2009). Evaluation of two Web-based alcohol interventions for mandated college students. Journal of Substance Abuse Treatment, 36(1), 65-74.

Faul, F., Erdfelder, E., Lang, A. G., \& Buchner, A. (2007). G*Power 3: a flexible statistical power analysis program for the social, behavioral, and biomedical sciences. Behavior Research Methods, 39(2), 175-191. 
GBD 2013 Risk Factors Collaborators. (2015). Global, regional, and national comparative risk assessment of 79 behavioural, environmental and occupational, and metabolic risks or clusters of risks in 188 countries, 1990-2013: a systematic analysis for the Global Burden of Disease Study 2013. Lancet, 386(10010), 2287-2323.

Gmel, G., Kuendig, H., Notari, L., \& Gmel, C. (2016). Suchtmonitoring Schweiz: Konsum von Alkohol, Tabak und illegalen Drogen in der Schweiz im Jahr 2015 [Addiction monitoring Switzerland: use of alcohol, tobacco and illegal drugs in Switzerland, data 2015] Lausanne: Sucht Schweiz.

Gmel, G., Venzin, V., Marmet, K., Danko, G., \& Labhart, F. (2012). A quasi-randomized group trial of a brief alcohol intervention on risky single occasion drinking among secondary school students. International Journal of Public Health, 57(6), 935-944.

Gollwitzer, P. M., \& Sheeran, P. (2006). Implementation intentions and goal achievement: a metaanalysis of effects and processes. Advances in Experimental Social Psychology, 38, 69119.

Haug, S., Kowatsch, T., Castro, R. P., Filler, A., \& Schaub, M. P. (2014). Efficacy of a web- and text messaging-based intervention to reduce problem drinking in young people: study protocol of a cluster-randomised controlled trial. BMC Public Health, 14, 809.

Haug, S., Paz Castro, R., Filler, A., Kowatsch, T., Fleisch, E., \& Schaub, M. P. (2014). Efficacy of an internet and SMS-based integrated smoking cessation and alcohol intervention for smoking cessation in young people: study protocol of a two-arm cluster randomised controlled trial. BMC Public Health, 14(1), 1140.

Haug, S., Paz Castro, R., Kowatsch, T., Filler, A., Dey, M., \& Schaub, M. P. (2017). Efficacy of a web- and text messaging-based intervention to reduce problem drinking in adolescents: Results of a cluster-randomized controlled trial. Journal of Consulting and Clinical Psychology, 85(2), 147-159. 
Haug, S., Schaub, M. P., Salis Gross, C., John, U., \& Meyer, C. (2013). Predictors of hazardous drinking, tobacco smoking and physical inactivity in vocational school students. $B M C$ Public Health, 13, 475.

Haug, S., Schaub, M. P., Venzin, V., Meyer, C., \& John, U. (2013). Efficacy of a text messagebased smoking cessation intervention for young people: a cluster randomized controlled trial. Journal of Medical Internet Research, 15(8), e171.

Heffner, J. L., Kealey, K. A., Marek, P. M., Bricker, J. B., Ludman, E. J., \& Peterson, A. V., Jr. (2016). Proactive telephone counseling for adolescent smokers: Comparing regular smokers with infrequent and occasional smokers on treatment receptivity, engagement, and outcomes. Drug and Alcohol Dependence, 165, 229-235.

Inchley, J., Currie, D., Young, T., Samdal, O., Torsheim, T., Augustson, L., . . Barnekow, V. (2016). Health behaviour in school-aged children (HBSC) study: International report from the 2013/2014 survey. Copenhagen: World Health Organization (WHO) Regional Office for Europe.

Kahler, C. W., Spillane, N. S., \& Metrik, J. (2010). Alcohol use and initial smoking lapses among heavy drinkers in smoking cessation treatment. Nicotine and Tobacco Research, 12(7), 781-785.

Laird, N. M., \& Ware, J. H. (1982). Random-effects models for longitudinal data. Biometrics, 38(4), 963-974.

Lippke, S., Ziegelmann, J. P., Schwarzer, R., \& Velicer, W. F. (2009). Validity of stage assessment in the adoption and maintenance of physical activity and fruit and vegetable consumption. Health Psychology, 28(2), 183-193.

Mason, M., Mennis, J., Way, T., Zaharakis, N., Campbell, L. F., Benotsch, E. G., . . King, L. (2016). Text Message Delivered Peer Network Counseling for Adolescent Smokers: A Randomized Controlled Trial. Journal of Primary Prevention, 37(5), 403-420. 
Mason, M., Ola, B., Zaharakis, N., \& Zhang, J. (2015). Text messaging interventions for adolescent and young adult substance use: a meta-analysis. Prevention Science, 16(2), 181188.

McAlister, A. L., Perry, C. L., \& Parcel, G. S. (2008). How Individuals, Environments, and Health Behaviour Interact: Social Cognitive Theory In K. Glanz, B. K. Rimer, \& K. Viswanath (Eds.), Health Behavior and Health Education: Theory, Research, and Practice. San Francisco: Jossey-Bass.

Melzer, J., Rissling, J. K., \& Petermann, F. (2015). [Influence of Nonverbal Intelligence and Migration Background on Language Abilities]. Gesundheitswesen, 77(10), 793-798. doi:10.1055/s-0035-1564148

Paz Castro, R., Haug, S., Kowatsch, T., Filler, A., \& Schaub, M. P. (2017). Moderators of outcome in a technology-based intervention to prevent and reduce problem drinking among adolescents. Addictive Behaviors, 72, 64-71.

Pbert, L., Farber, H., Horn, K., Lando, H. A., Muramoto, M., O'Loughlin, J., . . American Academy of Pediatrics, J. B. R. C. o. E. T. C. (2015). State-of-the-art office-based interventions to eliminate youth tobacco use: the past decade. Pediatrics, 135(4), 734-747.

Perkins, H. W. (2002). Social norms and the prevention of alcohol misuse in collegiate contexts. Journal of Studies on Alcohol, 63(14), 164-172.

Perkins, H. W. (2003). The Social Norms Approach to Preventing School and College Age Substance Abuse: A Handbook for Educators, Counselors, and Clinicians. (First ed.). San Francisco: Jossey-Bass.

Pocock, S. J. (1994). Clinical trials: a practical approach. Chichester: Wiley \& Sons.

Prince, M. A., Reid, A., Carey, K. B., \& Neighbors, C. (2014). Effects of normative feedback for drinkers who consume less than the norm: Dodging the boomerang. Psychology of Addictive Behaviors, 28(2), 538-544. 
Sayette, M. A., Martin, C. S., Wertz, J. M., Perrott, M. A., \& Peters, A. R. (2005). The effects of alcohol on cigarette craving in heavy smokers and tobacco chippers. Psychology of Addictive Behaviors, 19(3), 263-270.

Schwarzer, R. (2008). Modeling health behavior change: How to predict and modify the adoption and maintenance of health behaviors. Applied Psychology: An International Review, 57(1), $1-29$.

Schwarzer, R., Schuz, B., Ziegelmann, J. P., Lippke, S., Luszczynska, A., \& Scholz, U. (2007). Adoption and maintenance of four health behaviors: theory-guided longitudinal studies on dental flossing, seat belt use, dietary behavior, and physical activity. Annals of Behavioral Medicine, 33(2), 156-166.

SRNT Subcommittee on Biochemical Validation. (2002). Biochemical verification of tobacco use and cessation. Nicotine and Tobacco Research, 4, 149-159.

Stanton, A., \& Grimshaw, G. (2013). Tobacco cessation interventions for young people. The Cochrane database of systematic reviews, 8, CD003289.

Sussman, S., \& Sun, P. (2009). Youth tobacco use cessation: 2008 update. Tobacco Induced Diseases, 5, 3.

The ESPAD group. (2015). ESPAD Report 2015. Results from the European School Survey Project on Alcohol and Other Drugs. Lisbon: European Monitoring Centre for Drugs and Drug Addiction (EMCDDA).

Tworek, C., Yamaguchi, R., Kloska, D. D., Emery, S., Barker, D. C., Giovino, G. A., . . Chaloupka, F. J. (2010). State-level tobacco control policies and youth smoking cessation measures. Health Policy, 97(2-3), 136-144.

Van Buuren, S. (2012). Flexible Imputation of Missing Data. Boca Raton: CRC Press.

Van Zundert, R. M., \& Engels, R. C. (2009). Parental factors in association with adolescent smoking relapse. European Addiction Research, 15(4), 209-215. 
Van Zundert, R. M., Kuntsche, E., \& Engels, R. C. (2012). In the heat of the moment: alcohol consumption and smoking lapse and relapse among adolescents who have quit smoking. Drug and Alcohol Dependence, 126(1-2), 200-205.

Waller, G., Willemse, I., Genner, S., Suter, L., \& Süss, D. (2016). JAMES: Jugend, Aktivitäten, Medien - Erhebung Schweiz [JAMES: Youth, Activity, Media - Data Switzerland]. Zurich: Zürcher Hochschule für angewandte Wissenschaften.

Werch, C. E., \& Owen, D. M. (2002). Iatrogenic effects of alcohol and drug prevention programs. Journal of Studies on Alcohol, 63(5), 581-590.

Whittaker, R., McRobbie, H., Bullen, C., Rodgers, A., \& Gu, Y. (2016). Mobile phone-based interventions for smoking cessation. Cochrane Database of Systematic Reviews, 4 , CD006611. 
Table 1: Baseline characteristics of study sample. Values represent n (\%) unless stated otherwise.

\begin{tabular}{ccccc}
\hline MCT & MCT + & Total & $p$ \\
$\mathrm{n}=741$ & $\mathrm{n}=730$ & $\mathrm{~N}=1471$ & \\
\hline
\end{tabular}

Gender $^{\mathrm{a}, \mathrm{b}}$

Male

Female

Age, $M(S D)^{\mathrm{c}}$

Immigration background ${ }^{\text {a,d }}$

No immigration background

One parent born outside Switzerland

Both parents born outside Switzerland

Hours of extracurricular moderate to vigorous

physical activity per week, $M(S D)^{\mathrm{f}, \mathrm{g}}$

Number of alcoholic drinks consumed per week, $M(S D)^{\mathrm{c}}$

Number of alcoholic drinks consumed on heaviest drinking occasion, $M(S D)^{\mathrm{c}}$

Binge drinking ${ }^{a, b}$

No

Yes

Tobacco smoking status ${ }^{a, b}$

Daily smoker

Occasional smoker

Number of cigarettes smoked per day (CPD),

$M(S D)^{\mathrm{f}, \mathrm{g}}$

Stage of change a,e

No intention to quit

Considering quitting

Serious intention to quit

Previous quit attempts ${ }^{\text {a,e }}$

None

One

Two or more
.19

$279(37.7) \quad 299(41.0) \quad 578(39.3)$

$462(62.3) \quad 431(59.0) \quad 893(60.7)$

$18.9(3.6) \quad 18.4(2.5) \quad 18.6(3.1) \quad<.01$

.014

$331(44.7) \quad 372(51.0) \quad 703(47.8)$

$167(22.5) \quad 167(22.9) \quad 334(22.7)$

$243(32.8) \quad 191(26.2) \quad 433(29.5)$

$3.2(3.4) \quad 3.8(3.8) \quad 3.5(3.6) \quad<.01$

$9.8(12.9) \quad 10.0(11.1) \quad 10.3(18.0) \quad .32$

$\begin{array}{llll}7.9(8.3) & 7.6(8.3) \quad 8.1(8.4) \quad .18\end{array}$

.57

$249(33.6) \quad 235(32.2) \quad 484(32.9)$

$492(66.4) \quad 495(67.8) \quad 987(67.1)$

.05

$576(77.7) \quad 536(73.4) \quad 1112(75.6)$

$165(22.3) \quad 194(26.6) \quad 359(24.4)$

$10.4(7.2) \quad 9.8(7.5) \quad 10.1(7.3) \quad .08$

.41

$211(28.5) \quad 201(27.6) \quad 412(28.1)$

$435(58.8) \quad 417(57.3) \quad 852(58.0)$

$94(12.7) \quad 110(15.1) \quad 204(13.9)$

.55

$258(34.9) \quad 269(37.0) \quad 527(35.9)$

$328(44.3) \quad 302(41.5) \quad 630(42.9)$

$154(20.8) \quad 156(21.5) \quad 310(21.1)$ 
Note: $: \chi^{\mathrm{a}} \chi^{2}$ test, ${ }^{\mathrm{b}}(\mathrm{df}=1),{ }^{\mathrm{c}} U$ test, ${ }^{\mathrm{d}}(\mathrm{df}=2),{ }^{\mathrm{e}}(\mathrm{df}=3),{ }^{\mathrm{f}} t$ test, ${ }^{\mathrm{g}}(\mathrm{df}=1469)$ 
Table 2: Intervention effects for primary outcomes

\begin{tabular}{|c|c|c|c|c|c|c|c|c|}
\hline \multirow[b]{2}{*}{ Outcome } & \multirow[b]{2}{*}{ Baseline } & \multirow[b]{2}{*}{ Follow-up } & \multirow[b]{2}{*}{ Diff. } & \multicolumn{3}{|c|}{$\begin{array}{l}\text { Complete-case analysis } \\
\qquad(n=1116)\end{array}$} & \multicolumn{2}{|c|}{$\begin{array}{c}\text { Intention-to-treat analysis } \\
(n=1471)\end{array}$} \\
\hline & & & & test value & $p$ & Effect size $[95 \% \mathrm{CI}]$ & test value & $p$ \\
\hline 7-day abstinence, $\mathrm{n}(\%)$ & & & & 0.51 & .61 & $1.09[0.78,1.52]$ & 0.23 & .82 \\
\hline MCT & $0(100 \%)$ & $\begin{array}{l}77 / 552 \\
(13.9 \%)\end{array}$ & $13.9 \%$ & & & & & \\
\hline $\mathrm{MCT}+$ & $0(100 \%)$ & $\begin{array}{l}84 / 559 \\
(15.0 \%)\end{array}$ & $15.0 \%$ & & & & & \\
\hline Cigarettes per day, $M(S D)$ & & & & 0.04 & .97 & $0.00[-0.12 ; 0.12]$ & -0.09 & .93 \\
\hline MCT & $10.4(7.2)$ & $7.6(7.1)$ & -2.8 & & & & & \\
\hline $\mathrm{MCT}+$ & $9.8(7.5)$ & $7.1(6.6)$ & -2.7 & & & & & \\
\hline
\end{tabular}

Note. (Generalized) linear mixed models with group as a fixed factor and a random intercept for school classes. Parameters of (G)LMMs without control variables are displayed. Outcome for binary variables were follow-up values; for continuous variables differences from baseline to follow-up. Test values were $z$ values for binary outcomes; $t$ values for continuous outcomes. Effect sizes were Odds Ratio (OR) for binary outcomes; Cohen's $d$ for continuous outcomes. 
Table 3: Intervention effects for secondary outcomes

\begin{tabular}{|c|c|c|c|c|c|c|c|c|}
\hline \multirow[b]{2}{*}{ Outcome } & \multirow[b]{2}{*}{ Baseline } & \multirow[b]{2}{*}{ Follow-up } & \multirow[b]{2}{*}{ Diff. } & \multicolumn{3}{|c|}{$\begin{array}{l}\text { Complete-case analysis } \\
\qquad(n=1116)\end{array}$} & \multicolumn{2}{|c|}{$\begin{array}{l}\text { Intention-to-treat analysis } \\
\qquad(n=1471)\end{array}$} \\
\hline & & & & test value & $p$ & Effect size $[95 \% \mathrm{CI}]$ & test value & $p$ \\
\hline MCT & $0(100 \%)$ & $45 / 552(8.2 \%)$ & $8.2 \%$ & & & & & \\
\hline MCT+ & $0(100 \%)$ & $54 / 559(9.7 \%)$ & $9.7 \%$ & & & & & \\
\hline MCT & - & $205 / 544$ & $37.7 \%$ & & & & & \\
\hline & & $(37.7 \%)$ & & & & & & \\
\hline $\mathrm{MCT}+$ & - & $200 / 543$ & $36.8 \%$ & & & & & \\
\hline & & $(36.8 \%)$ & & & & & & \\
\hline $\mathrm{MCT}+$ & - & $272 / 552$ & $49.3 \%$ & & & & & \\
\hline & & $(49.3 \%)$ & & & & & & \\
\hline Alcoholic drinks/week, $M(S D)$ & & & & -0.44 & .74 & $0.02[-0.10 ; 0.14]$ & -1.09 & .28 \\
\hline MCT & $9.8(12.9)$ & $5.6(8.9)$ & -4.2 & & & & & \\
\hline $\mathrm{MCT}+$ & $10.0(11.1)$ & $5.2(7.9)$ & -4.8 & & & & & \\
\hline
\end{tabular}


Note. (Generalized) linear mixed models with group as a fixed factor and a random intercept for school classes. Parameters of (G)LMMs without control variables are displayed. Outcome for binary variables were follow-up values; for continuous variables differences from baseline to follow-up. Test values were $z$ values for binary outcomes; $t$ values for continuous outcomes. Effect sizes were Odds Ratios $(O R)$ for binary outcomes; Cohen's $d$ for continuous outcomes. 
Figure captions

Figure 1: Sample text messages on smoking cessation

Figure 2: Participants' progress through the trial

Figure 3: Number of drinks consumed on heaviest drinking occasion preceding baseline assessment moderating the intervention effect on seven-day point prevalence smoking abstinence at 6-months follow up 


\section{Stage-based smoking cessation messages}

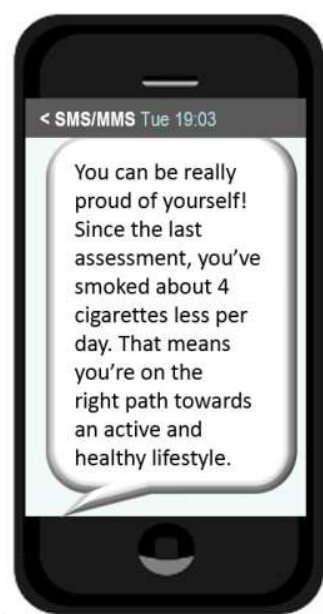

Non-Intender

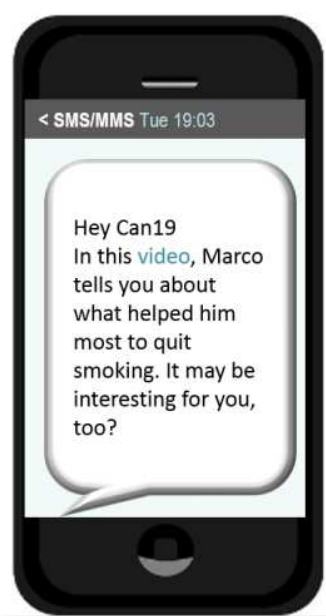

Intender

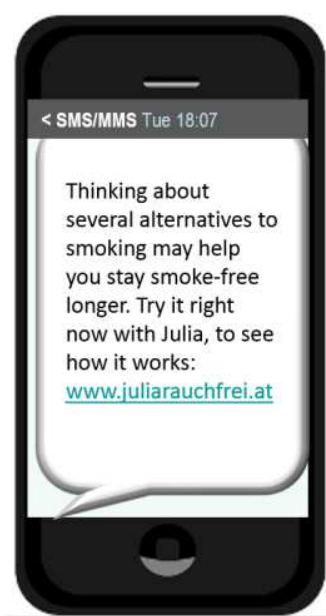

Actor

\section{Quiz question}
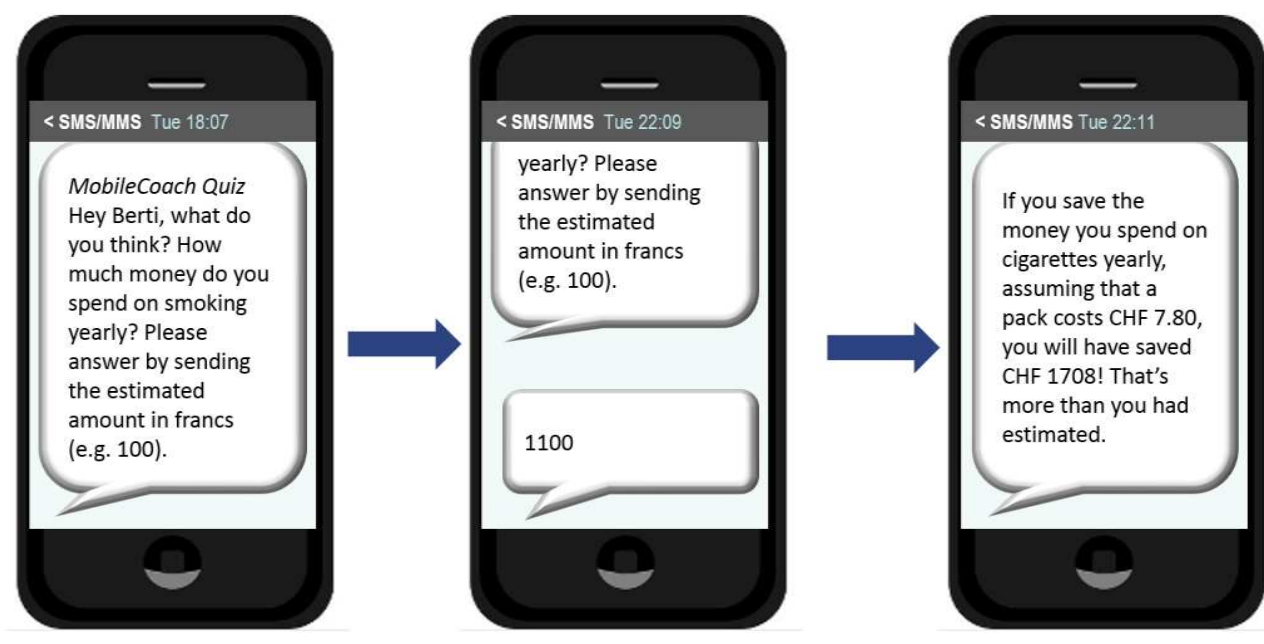

\section{Message contest}
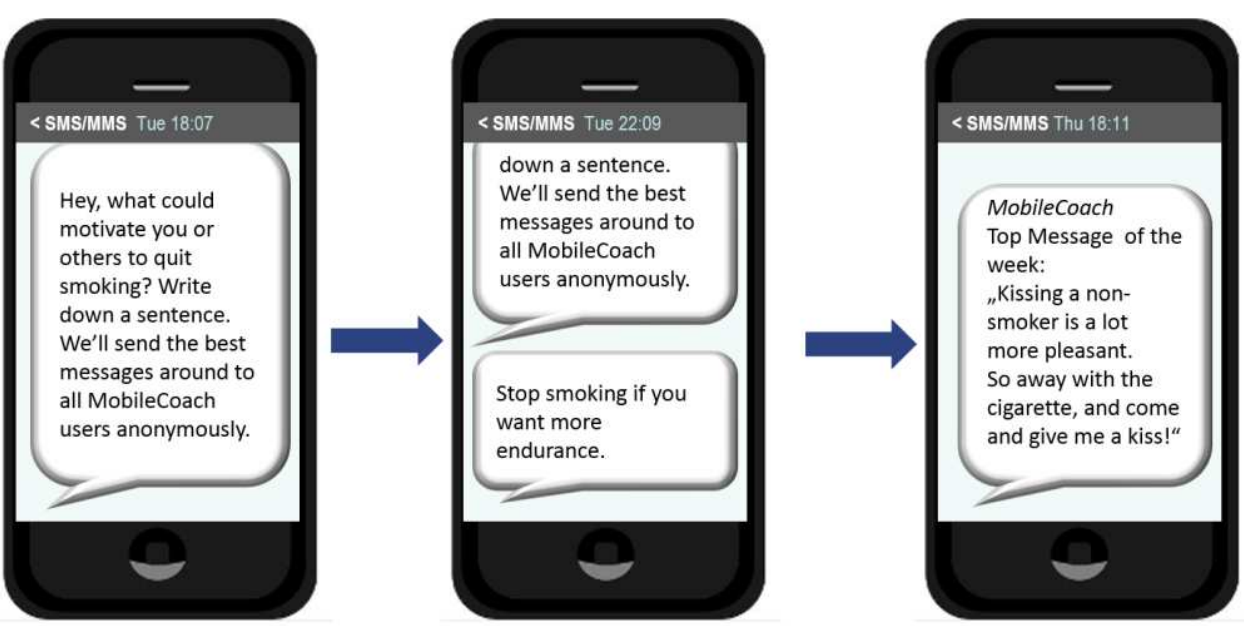


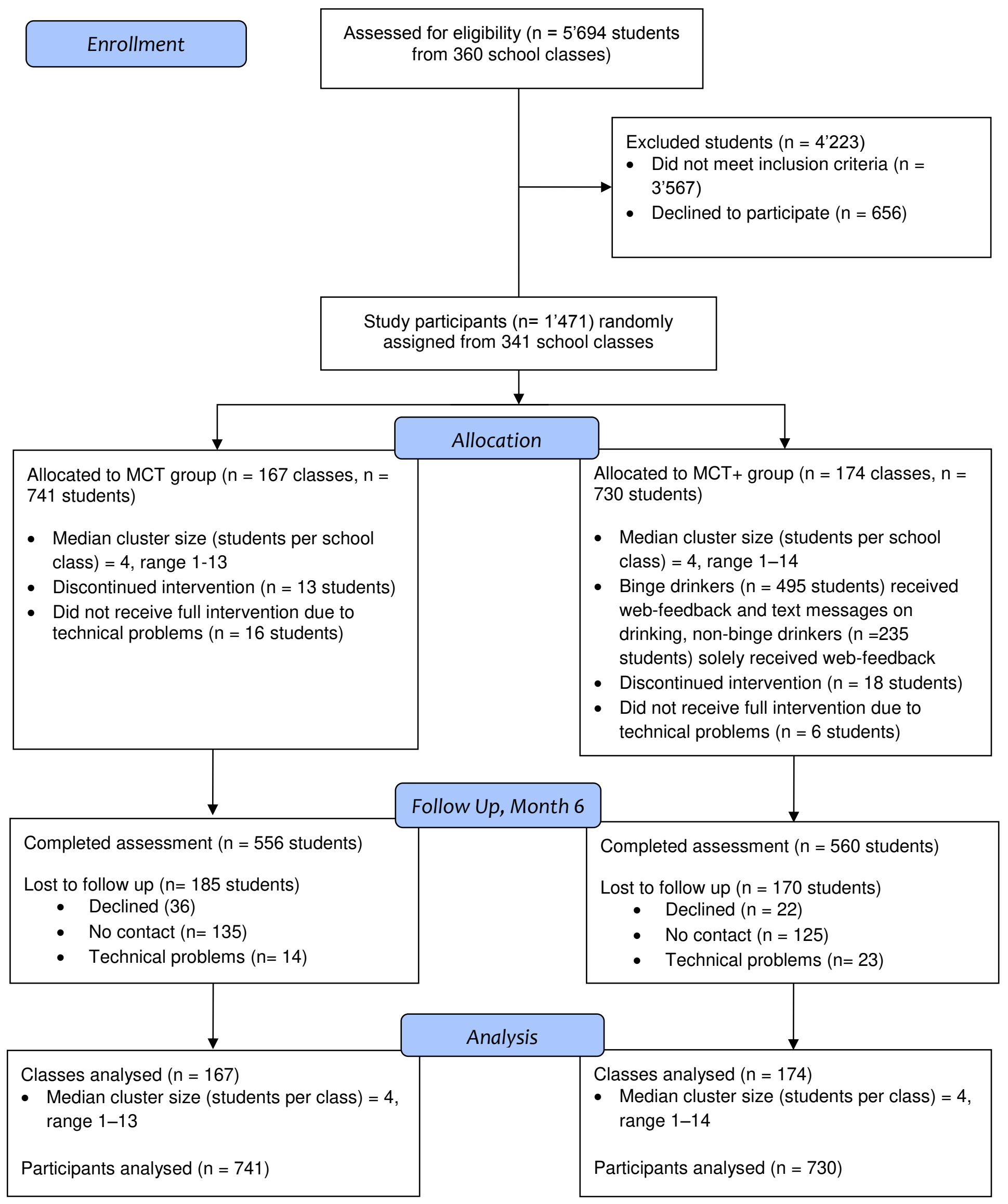




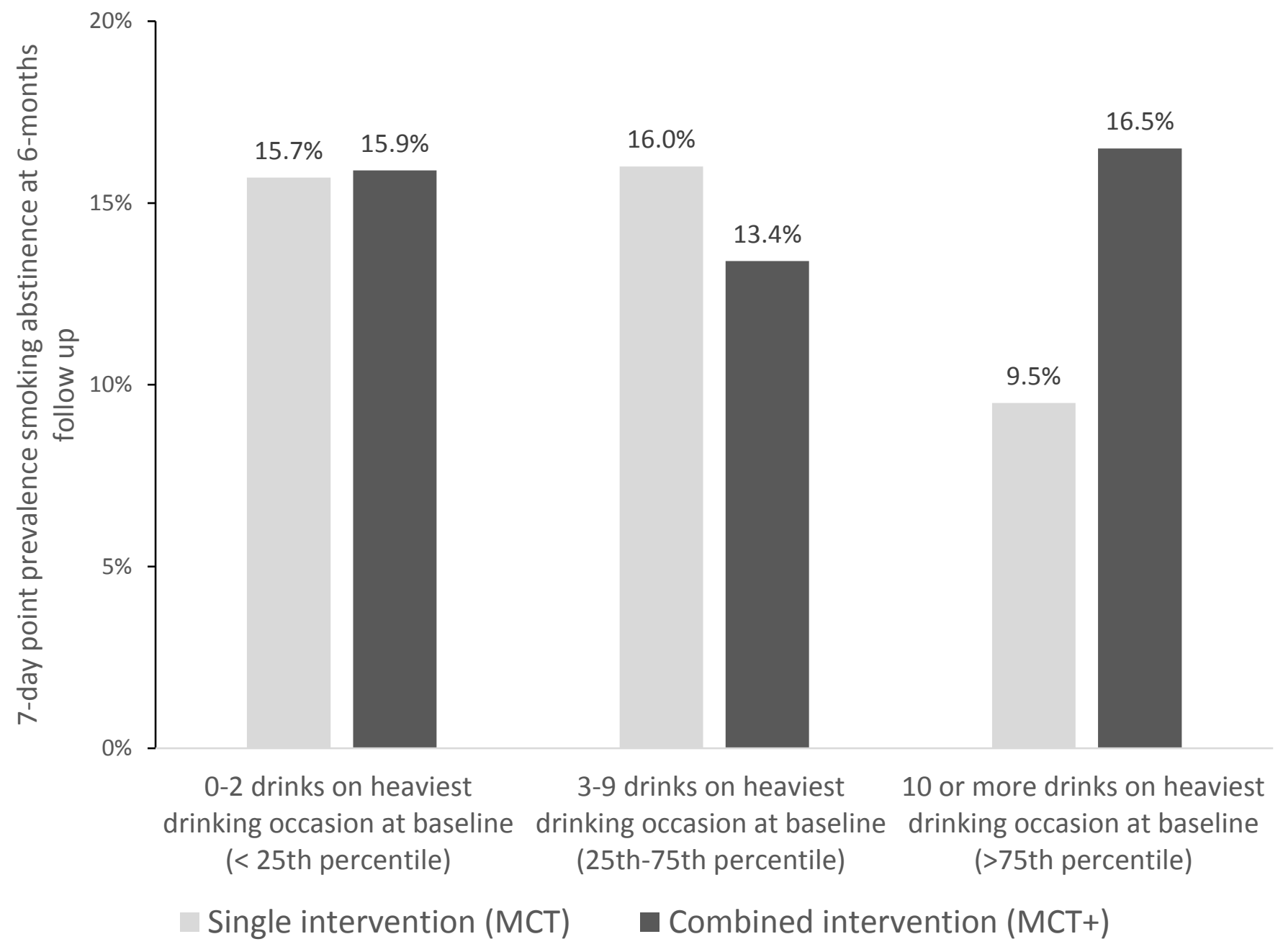

